\title{
(Supplementary) Impaired Function of Immunocytes in Diabetic Patients
}

\author{
Hideto SAKaI, MD \\ Department of Internal Medicine, School of Medicine, Tokai University, \\ Isehara City, Kanagawa-ken 259-11
}

It has been a matter of controversy whether diabetic patients without ketoacidosis are susceptible to infections. The purpose of the present study was to examine such susceptibility in diabetic patients and to elucidate the aberration of immunocytes in such patients.

Two hundred and seven patients were serially followed for 12 months at the Diabetes Clinic of the Tokai University Hospital. Thirty healthy adults served as controls. Only infections with both subjective and objective symptoms were counted in this study. Polymorphonuclear cells (PMN) and B lymphocytes (B cells) were separated from peripheral blood, and functions of these cells were analyzed as follows: (1) phagocytosis of PMN was determined by ingestion of either I-125 labelled zymosan or staphylococcus aureus (S. aureus). (2) intracellular reduction was detected by hot pyridine extraction of nitroblue tetrazolium (NBT) or intracellular killing of S. aureus. (3) in vitro production of IgG, IgA and IgM from pokeweed mitogen stimulated B cells was measured by immunofluorescent staining. (4) in vivo half-life of IgG was determined by injection of I-125 labelled deaggregated IgG into diabetic mice. (5) inhibitory effect of patients' sera as well as glucose and low density lipoprotein (LDL) on immunoglobulin production of B cells was analyzed in vitro. (6) recovery of functions of PMN and B cells was examined in patients followed by control of diabetes.

Following are the results obtained: (1) patients with poorly controlled diabetes as well as those with oral hypoglycemics or insulin showed a significant increase in the development of various types of infections. (2) levels of HgbAlc and serum LDL demonstrated a significant correlation with the emergence of infections. (3) reduction of NBT and intracellular killing of S. aureus in PMN were significantly impaired in poorly-controlled patients, while phagocytosis was not impaired in such patients. (4) in vitro production of immunoglobulins was significantly decreased in poorly-controlled patients, although serum levels of immunoglobulins were not changed in those patients. (5) in vivo half-life of IgG was significantly prolonged in diabetic mice. (6) both diabetic sera as well as a combination of glucose and LDL suppressed in vitro production of immunoglobulins from B cells. (7) functional recovery of PMN and B cells was observed in patients with successful control of diabetes, while in vitro addition of insulin did not show such improvement.

It is concluded that a combination of persistent hyperglycemia and hyperlipidemia lead to impaired functions of immunocytes. General improvement of diabetes is required for the recovery of immunological functions in such patients. 